\title{
The role of high-intensity focused ultrasound in ablation of atrial fibrillation and other cardiac arrhythmias
}

This article was published in the following Dove Press journal:

Research and Reports in Focused Ultrasound

15 December 2015

Number of times this article has been viewed

\author{
Asaf Danon' \\ Krishna Kumar Mohanan \\ Nair $^{2}$ \\ Jacob S Koruth ${ }^{3}$ \\ Andre d'Avila ${ }^{4}$ \\ Sheldon M Singh ${ }^{5}$ \\ 'Department of Cardiology, Lady \\ Davis Carmel Medical Center, Haifa, \\ Israel; '2Department of Cardiology, \\ Sree Chitra Tirunal Institute of \\ Medical Sciences and Technology, \\ Thiruvananthapuram, Kerala, India; \\ ${ }^{3}$ Helmsley Electrophysiology Center, \\ Mount Sinai School of Medicine, New \\ York, NY, USA; ${ }^{4}$ nstituto de Pesquisa \\ em Arritmia Cardiaca - Hospital \\ Cardiologico, Florianopolis, SC, Brazil; \\ ${ }^{5}$ Schulich Heart Program, Sunnybrook \\ Health Sciences Centre, University of \\ Toronto, Toronto, ON, Canada
}

Correspondence: Asaf Danon Department of Cardiology, Lady Davis Carmel Medical Center, 7 Michal Street, Haifa 34362I I, Israel

Email asdanon@gmail.com

\begin{abstract}
Atrial fibrillation is the most prevalent arrhythmia of the heart, originating usually from ectopic atrial activity of the pulmonary veins. Therefore, one of the treatment options is pulmonary vein isolation. Among the novel approaches to pulmonary vein isolation, highintensity focused ultrasound (HIFU) has been developed. The ultrasound energy can be focused on a specific area and would result in the formation of a lesion similar to that formed with radiofrequency (RF) ablation. Although preclinical studies were promising, clinical studies in patients resulted in lower efficacy and high complication rates in comparison to the standard ablation method, due to collateral damage. Using HIFU for epicardial approach during cardiac surgery and for extracorporeal ablation does seem to have a future role. In this review, we present the mechanism of HIFU lesion formation and the principal studies.
\end{abstract}

Keywords: atrial fibrillation, HIFU, high-intensity focused ultrasound, pulmonary vein isolation, epicardial, ablation

\section{Background}

Atrial fibrillation (AF) is the most prevalent and sustained arrhythmia worldwide. The incidence of AF has been increasing due to the increasing elderly population. ${ }^{1}$ Antiarrhythmic medications are only moderately effective in controlling AF, and their use is often limited by their side effects. Seminal work from Haissaguerre et al demonstrated that the predominant trigger for AF is ectopic activity originating in the pulmonary veins (PVs). ${ }^{2}$ Hence, strategies to electrically isolate the PVs from the remainder of the left atrium have evolved as the dominant nonpharmacologic strategy to treat AF.

The most commonly performed method for achieving electrical pulmonary vein isolation (PVI) involves the use of radiofrequency (RF) energy. Surgical methods utilizing RF energy have been using long probes, many fashioned as a clamp, to allow for the placement of contiguous lesions around the PV. Current percutaneous methods of isolation, however, require ablation in a point-by-point fashion along the ostium of the PVs. This procedure is operator-dependent, time-consuming, and results in a significant complication rate of $4 \%-5 \%$. Among the serious complications, stroke (up to $1 \%$ ), tamponade (1\%), atrial-esophageal fistula (AEF) $(0.1 \%)$, and PV stenosis $(<0.5 \%)$ are the most significant. ${ }^{3}$ Furthermore, clinical success is $\sim 70 \%-80 \%$ in 12 months. Therefore, a search for ablation strategy that may allow for easier PVI is ongoing.

Current novel approaches for PVI, frequently referred to as single-shot approaches, capitalize on the circular nature of the PV ostium. In essence, new approaches - both RF-based and non-RF-based - allow the delivery of energy sources using a circular or 
balloon-based catheter design. Some of the catheters that are currently used include cryoballoon (Medtronic, Minneapolis, MN, USA), which freezes the tissue; high-intensity focused ultrasound (HIFU; ProRhythm, Ronkonkoma, NY, USA), which delivers ultrasound (US) energy; the HeartLight laser balloon (CardioFocus, Marlborough, MA, USA); nMARK, which delivers RF energy (Biosense Webster Inc., Diamond Bar, CA, USA). It is proposed that the circular catheter-based strategy may allow for easy isolation of the PVs in relatively short time by allowing for the placement of contiguous lesions around the circumference of the PV. Furthermore, it is thought that these approaches would be less operator-dependent.

HIFU is one of the several approaches available for PVI (Figure 1). The first ablation using this technology in humans was reported in $2000 .^{4}$ This article will review the mechanism and clinical use of HIFU for the management of arrhythmia, with special attention to AF.

\section{The mechanism of HIFU lesion formation}

US is a form of vibration energy that can cause thermal tissue injury. The vibration wave propagates and creates mechanical movement of particles within a medium, which is then converted to heat. Thus, tissue damage is due to thermal injury. Further tissue damage also results from the acoustic cavitation, a process of formation of microbubbles by the propagated US wave. The formation of bubbles might enhance the effect of HIFU by creating a nonabsorbable medium (raising the acoustic impedance). This effect may also result in reflection of the US wave and heating of the prefocused area. ${ }^{5}$ Engel et al used a wedge preparation of dog heart to investigate the ability of HIFU to create lesions. Thermal damage results in protein denaturation, vacuoles formation, mitochondrial fragmentation, and clamping and fragmentation of Z-band of myocytes. ${ }^{6}$ Of note, this damage is also seen in lesions caused by RF as well, except for intramyocardial vacuolization. ${ }^{7,8}$

Experimental studies have demonstrated that the lesion size with HIFU appears to be dose-dependent - that is, increasing the application time and the acoustic power results in deeper lesions. The lesion created by HIFU can be deep (up to $11 \mathrm{~mm}$ ) and wide. ${ }^{9}$ Unlike RF lesions, HIFU lesions are well demarked with a histologically normal tissue surrounding the ablated tissue (Figure 2). Remarkably, it has been demonstrated that one can target a certain location with the application of HIFU energy. For example, Engel et al were able to create midmyocardial lesions in cardiac tissue while ensuring that the epi- and endocardium were intact. This property is in contrast to the RF lesions that decrease in efficacy with distance from the catheter. ${ }^{7,8}$ This property of HIFU may specifically be advantageous when ablating within the thick-walled left ventricle in patients with ventricular tachycardia, particularly those with mid- or epicardial circuits for ventricular tachycardia.

Another property of US energy that makes it advantageous for cardiac ablation is its ability to easily penetrate soft tissue. As such, US energy can produce focal lesions without the need to be in contact with the tissue. This property of HIFU makes it advantageous to other methods of ablating cardiac tissue. For example, proper contact is necessary for tissue injury to occur when RF or cryoablation energy
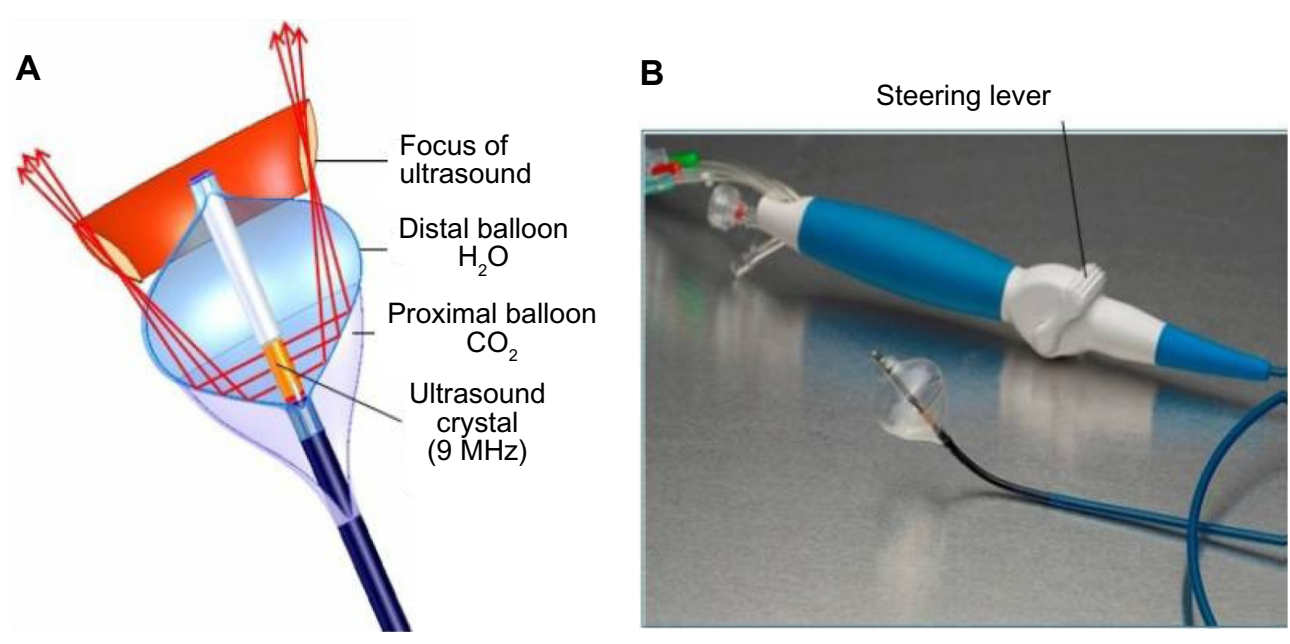

Figure I Schematic representation and photograph of the HIFU balloon.

Notes: (A) The noncompliant balloon, optimal for pulmonary vein ablation, is attached to a steerable catheter. An ultrasound crystal is located at the proximal part of the balloon. The balloon is filled with carbon dioxide and forms an interface allowing the reflection of the ultrasound beam. The acoustic power of the balloon is $45 \mathrm{~W}$. (B) The HIFU catheter with the inflated balloon. Reproduced from Schmidt B, Chun KRJ, Kuck KH, Antz M. Pulmonary vein isolation by high intensity focused ultrasound. Indian Pacing and Electrophysiology Journal. 2006;7(2): 126-133. ${ }^{32}$ Copyright (C) 2007 Schmidt et al.

Abbreviation: HIFU, high-intensity focused ultrasound. 
A

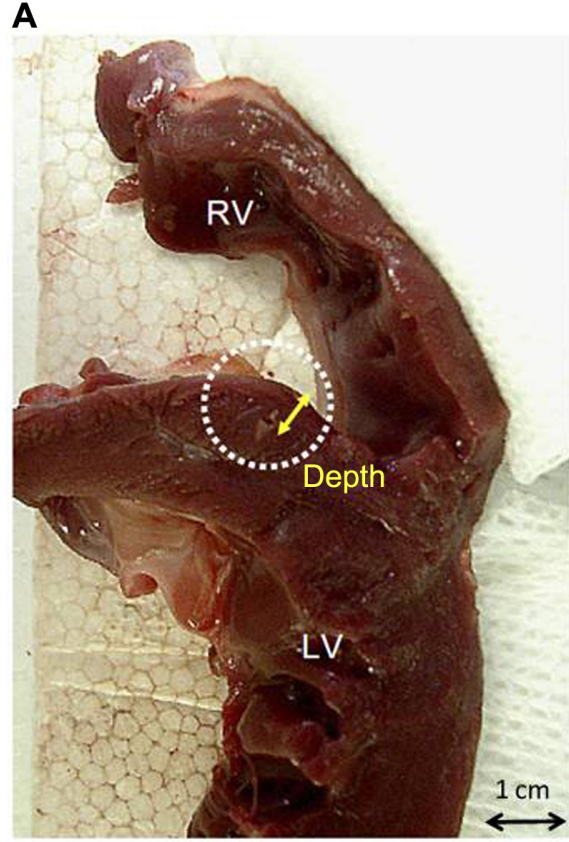

B

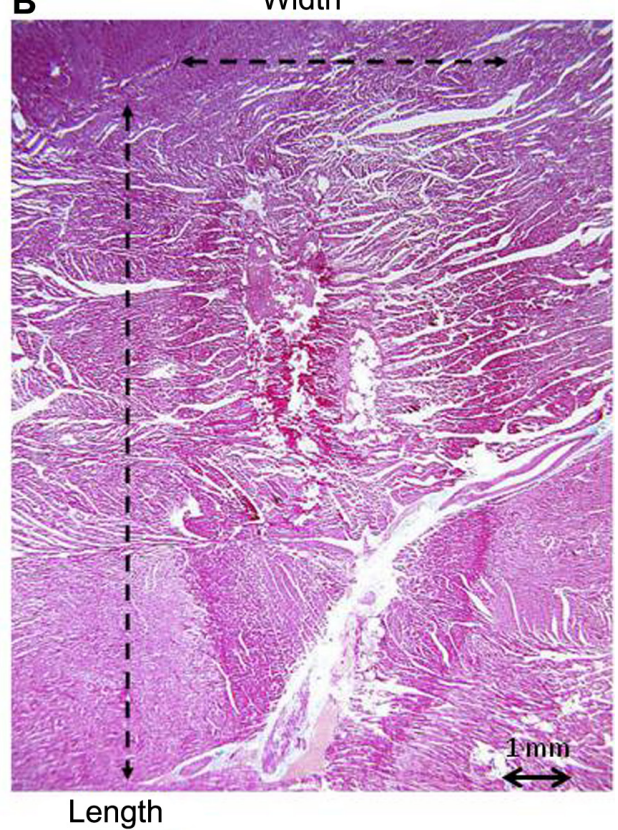

Figure 2 Lesion formation with HIFU.

Notes: HIFU lesion is shown in a model of a canine heart. (A) A deep, septal lesion is shown. Arrow denotes the deepness of the HIFU lesion. (B) A discrete necrotic lesion is shown with sparing of the surrounding tissue. Reprinted from the Journal of the American Society of Echocardiography; 2007;20; Otsuka R, Fujikura K, Abe Y, et al; Extracardiac ablation of the left ventricular septum in beating canine hearts using high-intensity focused ultrasound; 1400-I406; Copyright @ 2007 American Society of Echocardiography; Published by Elsevier; All rights reserved; with permission from Elsevier. ${ }^{32}$

Abbreviations: HIFU, high-intensity focused ultrasound; RV, right ventricle; LV, left ventricle.

is utilized. This property - produce lesions without the need to be in contact with the tissue - of HIFU makes it particularly advantageous in situations where challenging anatomy, based on the location of the tissue, and cardiac and respiratory motion impact the stability and consistency of catheter-tissue contact.

\section{Studies in animal models}

Zheng et al investigated the efficacy of HIFU in the canine heart. Lesion size was smaller than in in vivo model, suggesting that temperature cooling through blood flow is important in HIFU, similar to RF ablation. ${ }^{10}$ Okumura et al studied HIFU ablation of the right superior pulmonary vein (RSPV) in eight dogs using a steerable HIFU catheter (10 Fr balloon, diameter 24 mm; ProRhythm Inc.). Electrical PVI was confirmed in seven of eight PVs. The HIFU effect was inversely related to the distance, similar to RF energy. However, contact-independent lesion formation was demonstrated with HIFU, whereas this was not possible with RF energy. In addition, it was shown the HIFU lesions are much larger than RF lesions. Therefore, it was proposed that HIFU may result in faster and more effective tissue damage. ${ }^{11}$

One disadvantage of large rapid lesion formation is the potential for collateral injury (Figure 3 ). In the study by Zheng et al, phrenic nerve palsy was purposely induced. The authors proposed that a distance of $>7.5 \mathrm{~mm}$ between the HIFU exit and important structures is needed to prevent collateral damage. ${ }^{10}$ As we will explore in the following section, collateral injury may occur despite best intentions with HIFU.

\section{Safety and efficacy in human studies}

Several small, nonrandomized studies evaluated HIFU for PVI in humans. In 2006, Wong et al reported on the first patient undergoing PVI using the steerable HIFU balloon. ${ }^{12}$ Subsequently, Schmidt et al reported on the use of a steerable HIFU balloon in a cohort of 15 patients $^{13}$ - the success rate at 1 year with this approach was $75 \%$. However, the safety of this approach was questioned with two of the 15 patients $(13 \%)$ having permanent phrenic nerve palsy. Phrenic nerve injury occurred despite high-output pacing at the site of ablation to verify the absence of phrenic nerve capture, as well as pacing the phrenic nerve during ablation to observe loss of phrenic capture - strategies currently employed to protect the phrenic nerve during ablation procedure.

Schmidt et al went on to describe their experience 2 years later with the steerable HIFU balloon. ${ }^{14}$ Acute success was improved and procedure time was shortened dramatically. However, concerns about the high rate of procedural complications was again apparent with nearly one-third of all patients (four out of 15) suffering a major complication. 

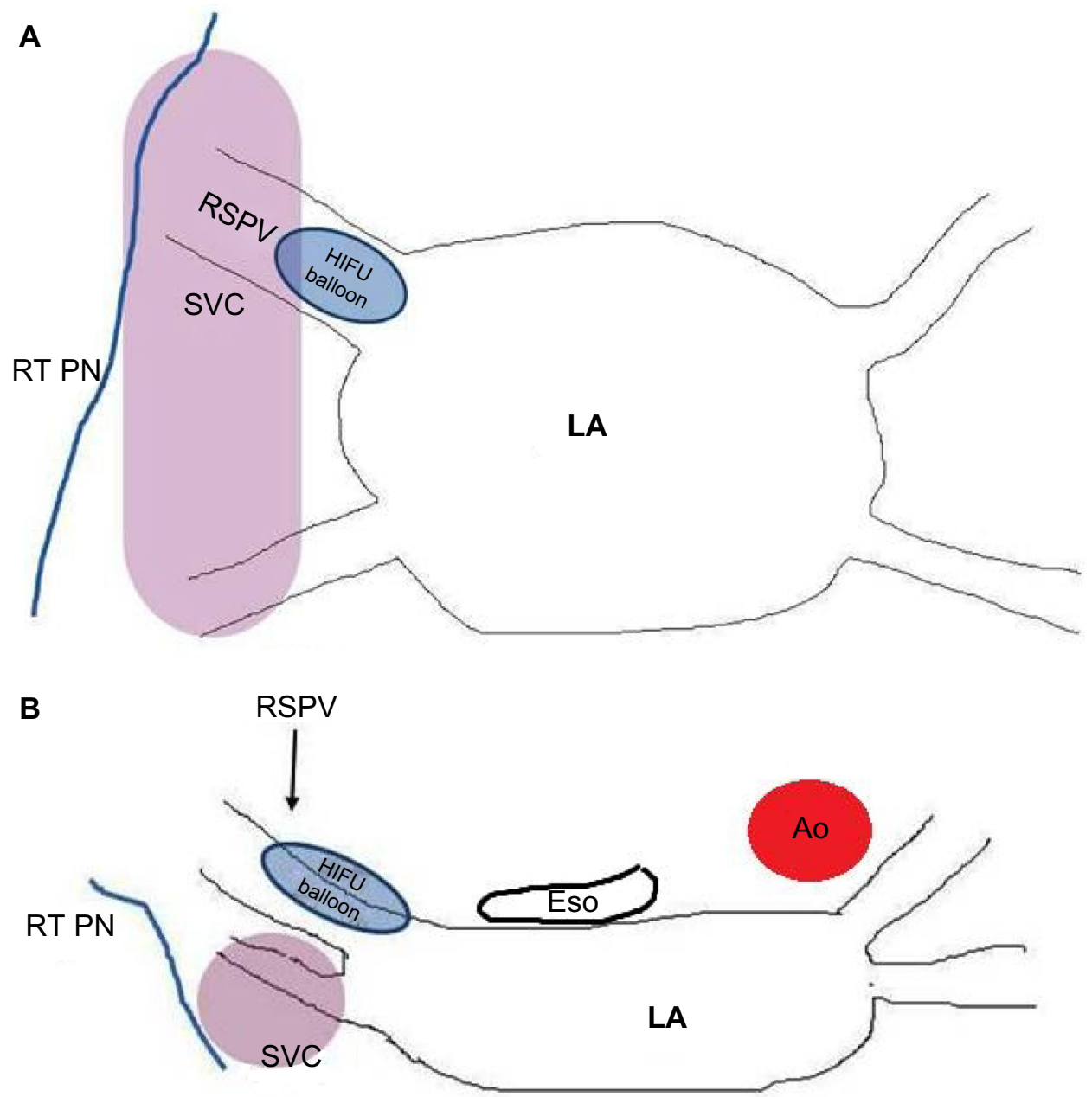

Figure 3 Location of structures prone to injury during balloon-based AF ablation.

Notes: Schematic image demonstrating anatomical relationship between the phrenic nerve and esophagus in relation to the pulmonary vein ostium. (A) Top, anteroposterior view and (B) top view.

Abbreviations: AF, atrial fibrillation; Ao, descending aorta; Eso, esophagus; HIFU, high-intensity focused ultrasound; LA, left atrium; RSPV, right superior pulmonary vein; RT PN, right phrenic nerve; SVC, superior vena cava.

The group also described a safety algorithm aimed at minimizing complications. ${ }^{15}$ This algorithm included $\leq 3$ complete HIFU applications per PV, early abortion when no effect was seen after $50 \%$ of programmed time or when esophageal temperature (ET) was $>40.0^{\circ} \mathrm{C}$, and the use of power modulation when an ET of $39.0^{\circ} \mathrm{C}$ was observed in order to reduce ablation temperature in the surrounding tissue. Touch-up RF ablation was used when PVI failed. ET monitoring and endoscopy were performed 2 days after ablation. Twenty-eight patients were included in the study. Of the 109 PVs, 84 were isolated with HIFU only. HIFU was aborted in nine due to high ETs. After a median follow-up of more than 2 years, $79 \%$ of the patients were free of $\mathrm{AF}$ without antiarrhythmic drugs or repeat ablation. However, this was achieved with the combination of HIFU and touch-up $\mathrm{RF}$ ablation or RF alone in $23 \%$ of the cases. Postprocedural endoscopy demonstrated a thermal lesion in two patients.
Two patients had persistent phrenic nerve palsies, one patient had an ischemic stroke, one patient experienced a pericardial effusion 48 days after ablation, one patient had fatal AEF 31 days after ablation, and one had an unexplained death 49 days after ablation. The study was prematurely stopped after the lethal case of AEF. The high complication rate (in all, 23\% experienced major complications) led the authors to conclude that the safety algorithm failed to prevent lethal complications.

Improving the safety algorithm suggested by Neven et a ${ }^{15}$ is difficult to perform. One may use power management (PM) in advance regardless of the esophageal power, which is not considered to be an ideal predictor of AEF. However, given the reduced efficacy with the current protocol, it is expected that further power reduction will decrease the rate of successful PVI much below the rates achieved by $\mathrm{RF}$ ablation and other methods. The same is expected with 
reduced power. Another option could be cover all but the US focus area (Figure 1) with a gas-filled balloon that will prevent energy delivery toward the phrenic nerve and other structures. However, the efficacy may be decreased, and it may still result in significant collateral damage.

\section{The HIFU balloon catheter}

The first-generation HIFU balloon was a nonsteerable overthe-wire device. The energy delivery was circumferential around the balloon equator. Saliba et al reported on 33 cases treated with HIFU balloon catheters. ${ }^{16}$ Success rate was only $40 \%$. Large or funnel-shaped PV ostium and early branching accounted for the low success rate. A second-generation balloon was developed with a steerable catheter and a forwardprojecting focused US beam. ${ }^{17}$ The catheter design has two balloons - the distal balloon containing the US crystal while the proximal balloon is filled with carbon dioxide and thus enables the forward projection of US wave (Figure 1). This design was thought to be more suitable for antral PVI rather than ablation within the vein itself, which may result in PV stenosis and phrenic nerve injury.

For PV ablation using HIFU, a double transeptal puncture is required, including $16.5 \mathrm{~F}$ sheath for the HIFU catheter. A second 8F sheath for the circular (lasso) catheter is positioned inside the PV to be ablated. Additional electrodes are positioned at the discretion of the operator but usually include at least a decapolar catheter in the coronary sinus. Different sizes of HIFU, including diameters of 24, 27, and $32 \mathrm{~mm}$, balloons are available. The choice is made according to the size of the PVs, measured usually in preoperative cardiac tomography (CT). HIFU application time varies between 40 and 90 seconds and depends on the balloon size (40, 60 , and 90 seconds for balloon size of 24, 27, and $32 \mathrm{~mm}$, respectively). Ideally, the balloon should be aligned with the axis of the PV. ${ }^{14}$

\section{Limitations of balloon technique}

A major limitation of any balloon-based ablation strategy is variability of the PV anatomy. For example, a large common left PV has been reported in up to $20 \%$ of patients undergoing AF ablation and right middle PV in $\sim 15 \%$ of patients. ${ }^{18}$ These variations create a major technical difficulty for the operator. Another limitation is collateral, and in particular permanent, injury to extracardiac tissue such as the phrenic nerve and esophagus. Phrenic nerve palsy may result in dyspnea and is difficult to overcome. Phrenic nerve injury may result from placing the HIFU balloon too distal in the vein. Although methods to minimize phrenic nerve injury have been proposed, phrenic nerve palsy remains significant with the use of the HIFU balloon. In contrast to ablation using cryoballoon, the phrenic nerve palsy caused by HIFU is rarely reversible. ${ }^{19}$ Esophageal injury may be fatal as was noted in early clinical studies. ${ }^{3}$

\section{Noninvasive ablation using HIFU}

Given the ability to direct the focus of US energy, it may be possible to utilize HIFU outside the vascular system. Indeed, extracorporeal HIFU ablation is widely used in oncology ${ }^{20,21}$ (prostate cancer, uterine fibroids, breast, and kidney tumors) and has been used for this purpose for more than 15 years. The US beam may be focused to a distant point without damaging the tissue between the transducer and the ablated tissue. Thus, extracorporeal ablation using HIFU may be advantageous by avoiding complications associated with vascular access and placement of catheters within the vascular system, such as cardiac perforation or stroke related to thrombus formation on the ablation equipment.

Strickberger et al created a model of extracardiac atrioventricular (AV) junction ablation. ${ }^{22}$ The ablation US transducer was attached to an imaging transducer and positioned within the thorax in a liquid medium. The AV junction area was identified with the US and a focused US ablation energy was applied during the diastole for only 30 seconds. All procedures resulted in AV block. Histologic sections showed a well-demarked area of acute inflammation with normal adjacent tissue. Otsuka et al similarly performed ventricular septal ablation, and suggested that this approach may be considered for treatment of hypertrophic obstructive cardiomyopathy. ${ }^{23}$ In these two studies, ${ }^{22,23}$ the ablation was performed through a thoracotomy due to attenuation of the US energy by the bony structures of the chest and the gas-rich lungs. However, extracorporeal HIFU can be used in the thorax as was shown for oncological states, mainly sub-diaphragmatic liver cancer. The most popular method is creating an artificial pleural effusion to displace the lung and improve the acoustic window. ${ }^{24,25}$ $\mathrm{Wu}$ et al created a pleural effusion by infusing saline and performed AV junction ablation successfully in all the 21 dogs. ${ }^{26}$ Rong et al used the same method to create ventricular septal lesions. In their study, $300 \mathrm{~mL}$ of saline was injected into the left pleura of 21 canines prior to the ablation, displacing the lung parenchyma and improving the acoustic window. During each HIFU application, lasting 20 seconds, mechanical ventilation was held to decrease the cardiac motion. ${ }^{27}$ In addition, meticulous care of the skin was needed, since any hair or dirt may cause US beam deflection and skin burns. 


\section{Epicardial ablation using HIFU}

Epicardial AF ablation has been used at the time of cardiac surgery as well as during percutaneous epicardial procedures. The original Maze-Cox procedure or its modification is currently used with reasonable outcomes. ${ }^{28}$ Davies et al

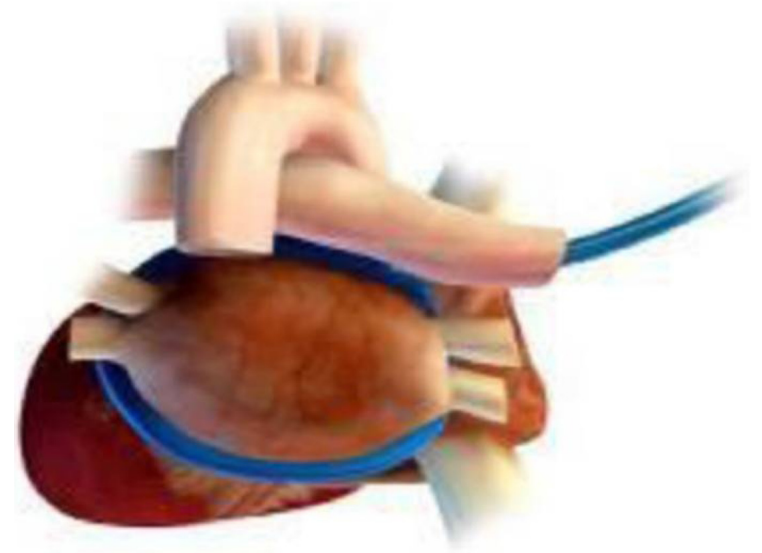

Figure 4 The Epicor epicardial HIFU system.

Notes: The epicardial HIFU system is placed around the four pulmonary veins to create the "box" lesion at the time of a cardiac surgical procedure for AF ablation. St Jude Medical is a trademark of St. Jude Medical, Inc. and its related companies. Reprinted with permission of St. Jude Medical, (C) 20I5. All rights reserved.

Abbreviations: HIFU, high-intensity focused ultrasound; $A F$, atrial fibrillation. recently reported on the use of the Epicor ablation system (St Jude Medical, Minneapolis, MN, USA; Figure 4) during surgical AF ablation procedures ${ }^{29}$ and confirmed its utility for surgical AF ablation with a 2-year freedom from $\mathrm{AF}$ of $81 \%$ for paroxysmal AF and $56 \%$ for persistent AF. Another group enrolled patients undergoing HIFU PVI during cardiac surgery. ${ }^{30}$ Each patient underwent electrophysiogical study with mapping 6 months after the HIFU ablation. Although $60 \%$ of the patients were free of AF at 6 months, only $38 \%$ had evidence of durable PVI.

The safety of epicardial HIFU application for ventricular tachycardia ablation (Figure 5) was studied by Koruth et al using a swine model ${ }^{31}$ In particular, the ability to focus the US beam and to avoid injury of adjacent structures was capitalized on, as HIFU application performed on top of coronary arteries did not result in significant coronary artery injury. Indeed, epicardial approaches to HIFU application may be important to consider for future use, as it may avoid collateral injury described with endocardial approaches and allow one to focus ablation to deeper sites. This approach may indeed prove beneficial for ablation of mid-myocardial and septal foci of ventricular tachycardia.

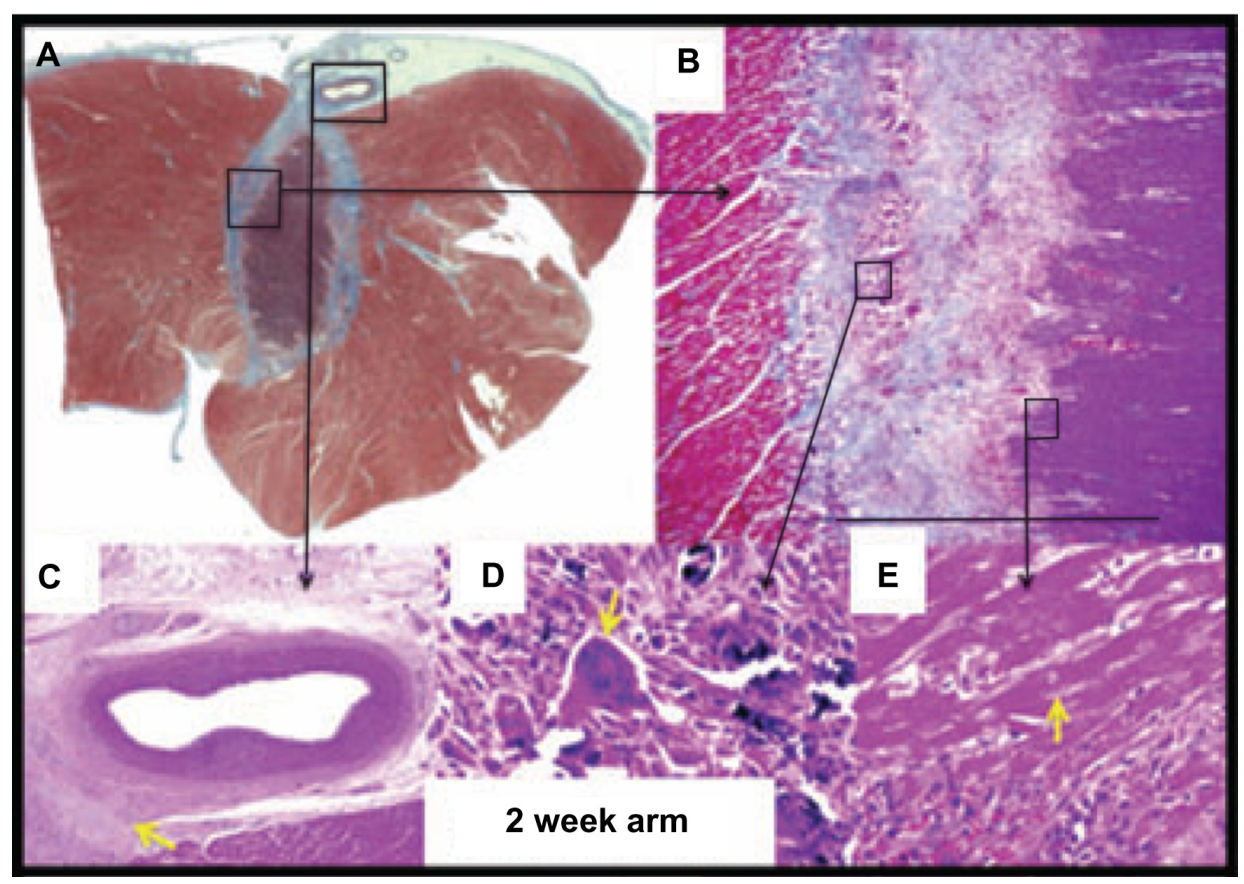

Figure 5 Epicardial ventricular ablation over a coronary artery.

Notes: (A) HIFU application on the epicardial aspect of the left ventricle over the left anterior descending artery. Note the absence of coronary artery injury. (B) Image (4X, Masson's trichrome [MA]) shows transition from viable myocytes (left) to fibrotic border with inflammation (middle) to necrotic myocytes (right); (C) image (4×, hematoxylineosin [HE]) shows LAD with adventitial fibrosis (yellow arrow); (D) image (40x, HE) shows calcification of necrotic myocytes with giant cell (yellow arrow); (E) image (40×, $\mathrm{HE}$ ) shows necrotic myocytes with nuclear loss (yellow arrow). Adapted from Koruth JS, Dukkipati S, Carrillo RG, et al. Safety and efficacy of high-intensity focused ultrasound atop coronary arteries during epicardial catheter ablation. Journal of Cardiovascular Electrophysiology. 20I I;22:1274-1280. ${ }^{31}$ Copyright (C) 20II Wiley Periodicals, Inc.

Abbreviations: HIFU, high-intensity focused ultrasound; LAD, left anterior descending artery. 


\section{Summary and future perspective}

Current transvenous HIFU ablation for AT carries a high complication rate. With the availability of other techniques (cryoballoon, circular RF catheters), it seems unlikely that this procedure will regain popularity. However, the utility of this approach may not be lost due to its potential for extracorporeal and epicardial ablation.

\section{Disclosure}

The authors report no conflicts of interest in this work.

\section{References}

1. ESC Committee for Practice Guidelines. Guidelines for the management of atrial fibrillation: The Task Force for the Management of Atrial Fibrillation of the European Society of Cardiology (ESC). Eur Heart J. 2010;31(19):2369-2429. doi:10.1093/eurheartj/ehq278.

2. Haissaguerre M, Jais P, Shah DC, et al. Spontaneous initiation of atrial fibrillation by ectopic beats originating in the pulmonary veins. $N$ Engl J Med. 1998;339:659-666.

3. Doppalapudi H, Yamada T, Kay GN. Complications during catheter ablation of atrial fibrillation: identification and prevention. Heart Rhythm. 2009;6:S18-S25.

4. Natale A, Pisano E, Shewchik J, et al. First human experience with pulmonary vein isolation using a through-the-balloon circumferential ultrasound ablation system for recurrent atrial fibrillation. Circulation. 2000;102:1879-1882.

5. Coussios CC, Farny CH, Ter HAAR G, et al. Role of acoustic cavitation in the delivery and monitoring of cancer treatment by high-intensity focused ultrasound (HIFU). Int J Hyperthermia. 2007;23:105-120.

6. Engel DJ, Muratore R, Hirata K, et al. Myocardial lesion formation using High-intensity Focused Ultrasound. J Am Soc Echocardiogr. 2006;19:932-937.

7. Nath S, Redick JA, Whayne JG, et al. Ultrastructural observations in the myocardium beyond the region of acute coagulation necrosis following radiofrequency catheter ablation. J Cardiovasc Electrophysiol. 1994;5:838-845.

8. Morady F. Radio-frequency ablation as treatment for cardiac arrhythmias. N Engl J Med. 1999;340:534-542.

9. He DS, Zimmer JE, Hynynen K, et al. Application of ultrasound energy for intracardiac ablation of arrhythmias. Eur Heart J. 1995;16:961-966.

10. Zheng M, Shentu W, Chen D, et al. High-intensity focused ultrasound ablation of myocardium in-vivo and instantaneous biological response. Echocardiography. 2014;31:1146-1153.

11. Okumura Y, Kolasa MW, Johnson SB, et al. Mechanism of tissue heating during high intensity focused ultrasound pulmonary vein isolation: implications for atrial fibrillation ablation efficacy and phrenic nerve protection. J Cardiovasc Electrophysiol. 2008;19:945-951.

12. Wong T, Markides V, Peters NS, et al. Anatomic left atrial circumferential ablation to electrically isolate pulmonary veins using a novel focused ultrasound balloon catheter. Heart Rhythm. 2006;3:370-371.

13. Schmidt B, Antz M, Ernst S, et al. Pulmonary vein isolation by highintensity focused ultrasound: first-in-man study with a steerable balloon catheter. Heart Rhythm. 2007;4:575-584.

14. Schmidt B, Chun KRJ, Metzner M, et al. Pulmonary vein isolation with high-intensity focused ultrasound: results from the HIFU 12F study. Europace. 2009;11:1281-1288.
15. Neven K, Schmidt B, Metzner A, et al. Fatal end of a safety algorithm for pulmonary vein isolation with use of high-intensity focused ultrasound. Circ Arrhythm Electrophysiol. 2010;3:260-265.

16. Saliba W, Wilber D, Packer D, et al. Circumferential ultrasound ablation for pulmonary vein isolation: analysis of acute and chronic failures. J Cardiovasc Electrophysiol. 2002;13:957-961.

17. Nakagawa H, Antz M, Wong $T$, et al. Initial experience using a forward directed, high-intensity focused ultrasound balloon catheter for pulmonary vein antrum isolation in patients with atrial fibrillation. J Cardiovasc Electrophysiol. 2007;18:136-144.

18. Mulder AA, Wijffils MC, Wever EF, Boersma LV. Pulmonary vein anatomy and long-term outcome after multi-electrode pulmonary vein isolation with phased radiofrequency energy for paroxysmal atrial fibrillation. Europace. 2011;13:1557-1561.

19. Metzner A, Chun KRJ, Neven K, et al. Long-term clinical outcome following pulmonary vein isolation with high-intensity focused ultrasound balloon catheters in patients with paroxysmal atrial fibrillation. Europace. 2010;12:188-193.

20. Al-Bataineh O, Jenne J, Huber P. Clinical and future applications of high intensity focused ultrasound in cancer. Cancer Treat Rev. 2012;38: 346-353.

21. Li T, Khokhlova T, Maloney E, et al. Endoscopic high-intensity focused US: technical aspects and studies in an in vivo porcine model (with video). Gastrointest Endosc. 2015;81:1243-1250.

22. Strickberger SA, Tokano T, Kluiwstra JUA, et al. Extracardiac ablation of the canine atrioventricular junction by use of high-intensity focused ultrasound. Circulation. 1999;100:203-208.

23. Otsuka R, Fujikura K, Abe Y, et al. Extracardiac ablation of the left ventricular septum in beating canine hearts using high-intensity focused ultrasound. J Am Soc Echocardiogr. 2007;20:1400-1406.

24. Fukuno H, Tamaki K, Urata M, et al. Influenced of an artificial pleural effusion technique $\mathrm{n}$ cardio-pulmonary function and autonomic activity. J Med Invest. 2007;54:48-53.

25. Shimada S, Hirota M, Beppu T, et al. A new procedure of percutaneous microwave coagulation therapy under artificial hydrothorax for patients with liver tumors in the hepatic dome. Surg Today. 2001;31: 40-44.

26. Wu W, Zhou Q, Zhu Q, et al. Noninvasive cardiac arrhythmia therapy using high-intensity focused ultrasound (HIFU) ablation. Inter J Card. 2013;166:e28-e30.

27. Rong S, Woo K, Zhou Q, et al. Septal ablation induced by transthoracic high-intensity focused ultrasound in canines. J Am Soc Echocardiogr. 2013;26:1228-1234.

28. Khargi K, Hutten BA, Lemke B, et al. Surgical treatment of atrial fibrillation: a systematic review. Euro J Cardiothor Surg. 2005;27: $258-265$.

29. Davies EJ, Bazerbashi S, Asopa S, et al. Long-term outcomes following high intensity focused ultrasound ablation for atrial fibrillation. J Card Surg. 2014;29:101-107.

30. Garcia R, Sacher F, Oses P, et al. Electrophysiogical study 6 months after Epicor ${ }^{\mathrm{TM}}$ high-intensity focused ultrasound atrial fibrillation ablation. J Interv Card Electrophysiol. 2014;41:245-251.

31. Koruth JS, Dukkipati S, Carrillo RG, et al. Safety and efficacy of high-intensity focused ultrasound atop coronary arteries during epicardial catheter ablation. J Cardiovasc Electrophysiol. 2011;22: 1274-1280.

32. Schmidt B, Chun KRJ, Kuck KH, Antz M. Pulmonary vein isolation by high intensity focused ultrasound. Indian Pacing and Electrophysiology Journal. 2006;7(2):126-133. 
Research and Reports in Focused Ultrasound

Dovepress

\section{Publish your work in this journal}

Research and Reports in Focused Ultrasound is an international, peerreviewed, open access online journal publishing original research, study protocols, reports, editorials, reviews and commentaries on all aspects of focused ultrasound technologies for the treatment of cancer and other diseases. The manuscript management system is completely

online and includes a very quick and fair peer-review system, which is all easy to use. Visit http://www.dovepress.com/testimonials.php to read real quotes from published authors.

Submit your manuscript here: http://www.dovepress.com/research-and-reports-in-focused-ultrasound-journal 